\title{
The Optimal Runner: a Control Problem with Phase Constraint
}

\author{
C. HAMBURGER
}

Assuming a simple biophysical model and using the Pontryagin maximum principle, we find the optimal strategy to run a race.

Key words: running, race, optimal velocity, phase constraint, maximum principle AMS subject classification: 49 B 10,49 B 36

\section{Introduction}

As a simple model describing the locomotion and the physiology of a runner the following system of differential equations has been proposed by J. B. Keller $[2,3]$ :

$$
\begin{aligned}
& v^{\prime}=\alpha f-\beta v, \quad 0 \leq f \leq F \text {, } \\
& E^{\prime}=\sigma_{\tau}^{2}-\gamma f v, \ldots . \quad . \quad E \geq 0 .
\end{aligned}
$$

Here time $t$ is the independent variable and $\alpha, \beta, \gamma, \sigma$ and $F$ are positive constants. The function $v$ is the velocity of the runner and $f$ is the force exerted by his or her muscular system, which cannot exceed a maximal value $F$. Equation (1) is Newton's equation of motion with a friction term which is proportional to the velocity.

The physiological energy for running is provided by the aerobic metabolism of carbohydrates, such as glucose circulating freely in the blood; and glycogen which is stored in the liver and muscles. In this process oxygen is consumed and carbon dioxide and water are produced. In a race the limiting factor in the provision of physiological energy is the oxygen, as carbohydrates are in abundant supply. Equation (2) describes the balance of disposable body oxygen $E$, which is being replenished at a constant rate $\sigma^{2}$ by respiration and consumed at a rate proportional to the power, $f v$, of the muscular action. Of course, $E$ is a non-negative quantity. We shall also refer to $E$ simply as the energy.

By an. appropriate choice of the units of length, time, force and oxygen we can fix $\alpha=\beta=\gamma=F=1$. We shall then assume that $0<\sigma<1$.

An alternative application of the above system is given by an electrically powered vehicle whose battery is recharged by mounted photovoltaic cells. :

C. Hamburger: Universitut Bonn, Math. Institut. Beringstr. 4, D - 53115 Bonn 
We shall be looking for the optimal strategy for the athlete to run a prescribed distance $D$ in least time $T$, or, what is easily seen to be an equivalent problem, to cover a maximal distance $D$ in a given amount of time $T$. The latter formulation is better suited to analysis as singular terms of the form $v^{-1}$ are avoided.

We are thus led to the following optimal control problem with phase constraint:

Problem : Given $\sigma \in] 0,1\left[, E_{0} \geq 0\right.$ and $T>0$, find state functions $v:[0, T] \rightarrow \mathbb{R}$, $E:[0, T] \longrightarrow[0,+\infty[$, and a control function $f:[0, T] \longrightarrow[0,1]$, satisfying the equations

$$
\begin{aligned}
& v^{\prime}=f-v \\
& E^{\prime}=\sigma^{2}-f v
\end{aligned}
$$

with initial values $v(0)=0, E(0)=E_{0}$, which maximize the integral

$$
\dot{D}=\int_{0}^{\mathrm{T}} v \mathrm{~d} t .
$$

Depending on the value of the allotted time $T$ in relation to the initial oxygen level $E_{0}$ and the respiratory rate $\sigma^{2}$, the solution of the problem is made up of one, 'two or three arcs, each chàracterized by either' running with maximal force $(f=1)$, running at constant speed $(v=$ const), or running with zero oxygen reserve $(E=0)$. In fact we have

Theorem : The above problem has a unique solution. There exist $T_{0}=T_{0}\left(\sigma, E_{0}\right)$ and $T_{\mathrm{c}} \cdot=T_{\mathrm{c}}\left(\sigma, E_{0}\right), ;$ with $0<T_{0}<T_{\mathrm{c}} \cdot \leq+\infty$, such that the solution is given, in the following three cases, $b y$.

(a) if $T \leq T_{0}$, then

$$
f(t)=1, \text { a.e., } E(t)>0 \quad \text { for } t \in] 0, \pi[
$$

(b) if $T_{0}<T \leq T_{c}$; then

$$
\begin{aligned}
& f(t)=1 \text { a.e., } E(t)>0 \\
& E(t)=0
\end{aligned}
$$

for $t \in] 0, T_{0}[$, for $t \dot{\epsilon}\left[T_{0}, T\right]$.

(c) if $T>T_{\mathrm{c}}$, then there are $v_{1}, T_{1}$ and $T_{2}$, with $0<T_{1}<T_{2}<T$, such that 


$$
\begin{aligned}
& f(t)=1 \text { a.e., } E(t)>0 \\
& v(t)=\text { const }=v_{1}, E(t)>0 \\
& E(t)=0
\end{aligned}
$$

Moreover $v_{1}$ and the final velocity $v(T)$ satisfy the relation

$$
v(T)=\frac{v_{1}^{2}+\sigma^{2}}{2 v_{1}},
$$

which determines $v_{1}, T_{1}$ and $T_{2}$ uniquely.

As is apparent from $(b), T_{0}$ is the amount of time which the runner can run with maximal force $(f=1)$ until he depletes his initial oxygen supply $E_{0}$. We let $v_{0}$ be the end velocity which he thus attains. We shall see that

$$
T_{\mathrm{c}}=T_{0}+\frac{1}{2} \log \frac{4 v_{0}^{2}}{v_{0}^{2}-\sigma^{2}}
$$

for $v_{0}>\sigma$, and $T_{\mathrm{c}}=+\infty$ for $v_{0} \leq \sigma$.

In the generic case, that is for $T$ and $E_{0}$ not too small, the optimal strategy is to run with maximal force $(f=1)$ until a certain velocity $v_{1}$ is reached, which is then maintained until the available body oxygen has been completely consumed. The last stretch of the race is run with zero oxygen reserve $(E=0)$ and decreasing velocity, the oxygen for running being supplied solely by respiration.

The optimality of running the major part of a long race with constant velocity is a generally accepted view. Our predicted strategy for the end part of the race, however, stands in marked contrast to the customary end sprints. End sprints may be accounted for by the unwillingness of the athlete to exhaust-his oxygen reserves before the finishing line is in sight. We can easily accomodate this psychological effect in our simple model by replacing the phase constraint $E \geq 0$ by a time-dependent phase constraint of the form $E(t) \geq E_{1}(t) \geq 0$, where $E_{1}(t)$ is a suitably decreasing function. It would be interesting to see what solutions are obtained under such a constraint.

In [3] J. B. Keller assumes the solution to the above problem to be of the form $f=1$ on $\left[0, T_{1}\right], E=0$ on $\left[T_{2}, T\right]$, and unconstrained on $] T_{1}, T_{2}\left[\right.$, for $0 \leq T_{1} \leq T_{2} \leq T$ and $T \geq T_{0}$. Using a variational procedure he then determines the values of $T_{1}$ and $T_{2}$, and the behaviour of $v$ on the interval $] T_{1}, T_{2}[$. However optimality for the proposed solution is not proven. In the present paper we present a rigorous proof of 
the theorem on the basis of the Pontryagin maximum principle for optimal control problems with phase constraint. It is a pleasure to acknowledge helpful discussions with F. H. Clarke and R. Klötzler.

A more realistic model for a runner has been developed by $H$. Behncke [1]. He obtains a similar solution from the Pontryagin maximum principle taking into account control constraints only.

\section{The Solution}

We now discuss in more detail the solution provided by the theorem. We consider the solution in $(a)$ and in $(b)$ as special cases of the solution in $(c)$ with $T_{1}=T_{2}=T$ and $T_{1}=T_{2}=T_{0}$ respectively. We shall see that the functions $T_{\mathrm{i}}=T_{\mathrm{i}}\left(\sigma, E_{0}, T\right)$ thus defined are piecewise analytic in $T$ with cusps at $T=T_{0} ; T_{c}$. In all cases $(a),(b)$ and (c) we let $v_{1}=v\left(T_{1}\right)=v\left(T_{2}\right)$.

In the sequel it will be convenient to choose the velocity $v$ as the parameter on the arcs which are run with maximal force $\left(f=1\right.$ on $\left.\left[0, T_{1}\right]\right)$ or with zero energy $\left(E=0\right.$ on $\left.\left[T_{2}, T\right]\right)$.

(i) If $f=1$ on $\left[0, T_{1}\right]$ and $v(0)=0$, then (3) has the solution

$$
v(t)=1-e^{-t}
$$

Since $v^{\prime}>0$, we can reparameterize this arc in terms of $v$. The energy expressed as a function of the velocity, $E=\epsilon(v)$, satisfies from (3) and (4) the differential equation

$$
\epsilon^{\prime}(v)=\frac{\sigma^{2}-v}{1-v}
$$

with initial value $\epsilon(0)=E_{0}$, so we obtain

$$
\epsilon(v)=E_{0}+v-\left(1-\sigma^{2}\right) \log \frac{1}{1-v} \quad(0 \leq v<1)
$$

We calculate the velocity $v_{0}$ which the runner attains after running with maximal force $(f=1)$ until his initial energy supply $E_{0}$ is depleted. From $\lim _{v \rightarrow 1} \epsilon(v)=-\infty$, and $\epsilon^{\prime}(v)>0$ for $0 \leq v<\sigma^{2}$ and $\epsilon^{\prime}(v)<0$ for $\sigma^{2}<v<1$, we see that there is precisely one velocity $v_{0}$ which satisfies $\epsilon\left(v_{0}\right)=0$, or, equivalently,

$$
E_{0}=\left(1-\sigma^{2}\right) \log \frac{1}{1-v_{0}}-v_{0}
$$


The time at which $v_{0}$ is attained is, from (7),

$$
T_{0}=\log \frac{1}{1-v_{0}}
$$

(ii) When $E=0$ on $\left[T_{2}, T\right]$, then (4) implies that $v \neq 0$ and $f=\sigma^{2} v^{-1}$. Inserting this value in (3) gives

$$
v^{\prime}=v^{-1}\left(\sigma^{2}-v^{2}\right)
$$

which has the solution

$$
v(t)=\left[\left(v_{1}^{2}-\sigma^{2}\right) e^{-2\left(t-T_{2}\right)}+\sigma^{2}\right]^{\frac{1}{2}}
$$

satisfying $v\left(T_{2}\right)=v_{1}$. From (12) we see that, on $\left[T_{2}, T\right], v \neq \sigma$, unless $v=$ const $=\sigma$. For $v \neq \sigma$, we have by (11) $v^{\prime} \neq 0$, and we can choose the velocity $v$ as parameter.

(iii) In case $(c), v=$ const $=v_{1}$ on $\left[T_{1}, T_{2}\right], E\left(T_{1}\right)=\epsilon\left(v_{1}\right)>0$, where $\epsilon$ is given by (8), and $E\left(T_{2}\right)=0$. Since, by (3) and (4), $f=v=v_{1}$ and $E^{\prime}=\sigma^{2}-v_{1}^{2}<0$, and since $\epsilon(v) \leq 0$ for $v_{0} \leq v<1$, we conclude that

$$
\sigma<v_{1}<v_{0} \text { and } T_{2}=T_{1}+\frac{\epsilon\left(v_{1}\right)}{v_{1}^{2}-\sigma^{2}} \text {. }
$$

We now proceed to calculate $v_{1}, T_{1}$ and $T_{2}$ in terms of $T, E_{0}$ and $\sigma$. Formulas (7) and (12) yield

$$
T_{1}=\log \frac{1}{1-v_{1}} \text { and } T=T_{2}+\frac{1}{2} \log \frac{v_{1}^{2}-\sigma^{2}}{v^{2}(T)-\sigma^{2}}
$$

Combining (5), (13) and (14), we obtain $T=\tau\left(v_{1}\right)$, for $\tau$ the function

$$
r(v):=\log \frac{1}{1-v}+\frac{\epsilon(v)}{v^{2}-\sigma^{2}}+\frac{1}{2} \log \frac{4 v^{2}}{v^{2}-\sigma^{2}}
$$

This function is seen to be a bijection $\left.\tau:] \sigma, v_{0}\right] \rightarrow\left[T_{\mathrm{c}},+\infty\left[\right.\right.$, since $\tau\left(v_{0}\right)=T_{\mathrm{c}}$ from (6) and (10), $\lim _{v \rightarrow \sigma} \tau(v)=+\infty$ and $\tau^{\prime}<0$. For $T>T_{\mathrm{c}}$, the quantity $v_{1}=\tau^{-1}(T)$ with $\sigma<v_{1}<v_{0}$ is therefore determined uniquely from $T, E_{0}$ and $\sigma$, as are $T_{1}$ and $T_{2}$.

(iv) It can be checked that, in all cases $(a),(b)$ and $(c), D$ is given by 


$$
D=\int_{0}^{\mathrm{T}} f \mathrm{~d} t-v(T)=T_{1}+v_{1}\left(T_{2}-T_{1}\right)+\sigma\left(T-T_{2}\right)+\sigma \log \frac{v(T)+{ }^{\prime \prime} \sigma}{v_{1}+\sigma}-v(T)
$$

We here insert, in case ( $a), T_{1}=T_{2}=T$ and $v_{1}=\vartheta(T)$ given by (7); in case $(b), T_{1}=$ $T_{2}=T_{0}, v_{1}=v_{0}$ as determined by (9) and $v(T)$ from (12); in case (c), $v_{1}=\tau^{-1}(T)$ with $\tau$ the function (15), $v(T)$ as defined by (5) and $T_{1}$ and $T_{2}$ given by (14) and (13) respectively.

\section{Proof of the Theorem}

The existence of a solution $(v, E) \in W^{1,1}\left([0, T], \mathbb{R} \times\left[0,+\infty[)\right.\right.$ with $f \in L^{1}([0, T],[0,1])$ to our problem follows easily from [4, p.373; Theorem $3($ iii $)]$.

We first note that the solution of (3), with $v(0)=0$, is

$$
v(t)=\int_{0}^{t} f(\tau) e^{\tau-t} \mathrm{~d} \tau
$$

whence we see that $0 \leq v<1$. We also infer from (16) that $v(t) \geq v\left(t_{1}\right) e^{t_{1}-t}$, for $t \geq t_{1}$, in particular that

$$
v\left(t_{1}\right)>0 \Rightarrow v(t)>0 \text { for all } t \in\left[t_{1}, T\right]
$$

We now apply the Pontryagin maximum principle for optimal control problems with phase constraints [4, p.234; Theorem 1]. For the Pontryagin function associated with our problem we find

$$
\begin{aligned}
H\left(v, E, f, p, q, \lambda_{0}\right) & =p(f-v)+q\left(\sigma^{2}-f v\right)+2 \lambda_{0} v \\
& =(p-q v) f+\text { terms not involving } f
\end{aligned}
$$

for which $H_{v}=-p-q f+2 \lambda_{0}$ and $H_{E}=0$. We therefore infer that there exist a number $\lambda_{0} \geq 0$, two functions $p(t)$ and $\cdot q(t)$, and a non-negative regular measure $\mu$ supported on the set $E^{-1}\{0\}$, not all zero, such that

$$
p(t)=\int_{\mathrm{t}}^{\mathrm{T}}\left(-p-q f+2 \lambda_{0}\right) \mathrm{d} \tau \text { and } q(t)=\int_{\mathrm{t}}^{\mathrm{T}} \mathrm{d} \mu=\mu([t, T])
$$


We conclude that

$$
p(T)=0 \text { and } q(T)=\mu(\{T\}) \geq 0 \text {, }
$$

that $p$ is absolutely continuous and satisfies the equation

$$
p^{\prime}=p+q f-2 \lambda_{0} \text { a.e. in }[0, T]
$$

whereas $q$ is positive, non-increasing, constant on every component of $\{t: E(t)>0\}$, continuous from the left, and the right limit $q(t+)$ exists at every point $t<T$ and satisfies $q(t) \geq q(t+)$.

We also infer from the Pontryagin maximum principle that for a.e. $t \in[0, T]$

$$
s(t)>0 \Rightarrow f(t)=1, s(t)<0 \Rightarrow f(t)=0,
$$

where we have introduced the switching function $s=p-q v$. The function $s$, like $q$, is continuous from the left and its right limit $s(t+)$ exists at every point. From $v \geq 0$ and $q(t) \geq q(t+)$, we deduce

$$
s(t) \leq s(t+)
$$

On $\{E>0\}$ we have from (19) and (3)

$$
s^{\prime}=p-2 \lambda_{0}+q v,
$$

whence we see that $s \in C^{1}$ on $\{E>0\}$.

On an interval where $f=0$ or where $q=$ const and $f=$ const, we can solve equations (19) and (3) obtaining

$$
p(t)=c_{1} e^{t}+2 \lambda_{0}-q f \text { and } v(t)=c_{2} e^{-t}+f
$$

and thus

$$
s(t)=2\left(\lambda_{0}-q f\right)+\alpha \cosh \left(t-t_{1}\right)+\beta \sinh \left(t-t_{1}\right)
$$

for arbitrary $t_{1}$ and suitable $\alpha, \beta \in \mathbb{R}$.

Lemma 1 : Let $E\left(t_{0}\right)=0$ with $0<t_{0} \leq T$. Then we cannot have $f=0$ in a left neighbourhood of $t_{0}$ or $f=1$ in a right neighbourhood of $t_{0}$. 
Proof : If $f=0$ in a left neighbourhood of $t_{0}$, then, by (4), $E(t)=\sigma^{2}\left(t-t_{0}\right)<0$ for $t<t_{0}$ in that neighbourhood, which is absurd.

We next show that $v\left(t_{0}\right) \geq \sigma^{2}$. For if $v\left(t_{0}\right)<\sigma^{2}$, then $v<\sigma^{2}$ in a neighbourhood of $t_{0}$ and, for $t<t_{0}$ in that neighbourhood, we have

$$
E(t)=\int_{\mathrm{t}}^{\mathrm{t}_{0}}\left(f v-\sigma^{2}\right) \mathrm{d} \tau \leq \int_{\mathrm{t}}^{\mathrm{t}_{0}}\left(v-\sigma^{2}\right) \mathrm{d} \tau<0
$$

which contradicts $E \geq 0$.

Suppose now that $f=1$ in a right neighbourhood of $t_{0}$. It then follows that, for $t>t_{0}$ in that neighbourhood,

$$
v(t)=v\left(t_{0}\right)+\int_{\mathrm{t}_{0}}^{\mathrm{t}}(1-v) \mathrm{d} \tau>v\left(t_{0}\right) \geq \sigma^{2} \text {, and hence, } E(t)=\int_{\mathrm{t}_{0}}^{\mathrm{t}}\left(\sigma^{2}-v\right) \mathrm{d} \tau<0,
$$

which cannot be true

Corollary 1 : The functions $q$ and $s$ vanish at the right end point:

$$
q(T)=s(T)=0
$$

Proof: The inequality $q(T)>0$ implies by (18), since $\mu$ has support on $E^{-1}\{0\}$, that $E(T)=0$. From (16) we see that $v(T)>0$, unless $v \equiv f \equiv 0$ which is absurd. Therefore $s(T)<0$, and since $s$ is continuous from the left, we have $s<0$ and, by (20), $f=0$ in a left neighbourhood of $T$. This stands in contradiction to Lemma 1

Corollary 2 : If $E\left(t_{0}\right)=0$ with $0<t_{0} \leq T$, then $s\left(t_{0}\right)=0$ and $s$ is continuous at $t_{0}$.

Proof : We can assume that $0<t_{0}<T$. Now if $s\left(t_{0}\right)<0$, then, since $s$ is continuous from the left, $s<0$, hence $f=0$, in a left neighbourhood of $t_{0}$. This cannot happen by Lemma 1. And if $s\left(t_{0} t\right)>0$, we have $s>0$ and $f=1$ in a right neighbourhood of $t_{0}$. Again, by Lemma 1, this is not possible.

Hence from (21) we have $0 \leq s\left(t_{0}\right) \leq s\left(t_{0}+\right) \leq 0$, and thus $s\left(t_{0}\right)=s\left(t_{0}+\right)=0$

Corollary 3 : The switching function $s$ is continuous.

Proof : The function $s$ is of class $C^{l}$ on $\{E>0\}$, continuous on $\left.\{t \in] 0, T\right]$ : 
$E(t)=0\}$ by Corollary 2 and continuous in 0, since $s=p-q v$ with $v(0)=0$

Lemma 2 : The switching function is non-negative: $s \geq 0$.

Proof : If we suppose to the contrary that $\{s<0\} \neq \emptyset$, then, by (24), we would have $s<0$ on an interval $] t_{1}, t_{2}\left[\right.$, for $0 \leq t_{1}<t_{2} \leq T$, with $s\left(t_{2}\right)=0$ and either $t_{1}=0$ or $s\left(t_{1}\right)=0$.

If $t_{1}=0$, then $v=f=0$ and $s=p$ on $\left[0, t_{2}\right]$, in particular $p\left(t_{2}\right)=s\left(t_{2}\right)=0$. Thus, from (22), we obtain

$$
s(t)=p(t)=2 \lambda_{0}\left(1-e^{t-t_{2}}\right) \geq 0
$$

for $t \in\left[0, t_{2}\right]$, in contradiction to our assumption that $s<0$ on $] 0, t_{2}[$.

And if $s<0$, and hence $f=0$, on an interval $] t_{1}, t_{2}\left[\right.$, with $s\left(t_{1}\right)=s\left(t_{2}\right)=0$, then, from (23), $s$ would be given by

$$
s(t)=-2 \lambda_{0}\left[\cosh \left(t-t_{1}\right)-1\right]+\beta \sinh \left(t-t_{1}\right)
$$

for $t \in\left[t_{1}, t_{2}\right]$. We see that $\beta$ is the derivative from the right of $s$ at $t_{1}$. Since $s\left(t_{1}\right)>s(t)$ for $t_{1}<t<t_{2}$, we must have $\beta \leq 0$. Equation (25) would then imply that $s\left(t_{2}\right)<0$, unless $\lambda_{0}=\beta=0$, but our assumption was $s\left(t_{2}\right)=0$ and $s<0$ on $] t_{1}, t_{2}[$.

Thus we have shown that $s \geq 0$

We now dispose of the case that $\lambda_{0}=0$. If $\lambda_{0}=0$, then (18) and (19) are solved by

$$
p(t)=-\int_{\mathrm{t}}^{\mathrm{T}} q(\tau) f(\tau) e^{t-\tau} \mathrm{d} \tau
$$

from which we see that $p \leq 0$. By Lemma 2, we cannot have $p(0)=s(0)<0$. Therefore $p(0)=0$, and, since the integrand in (26) is non-negative, it follows that $p \equiv 0$, and further, by Lemma 2 , that

$$
s=-q v \equiv 0 .
$$

Let $t_{0}=\sup \{t: v=0$ on $[0, t]\}$. If $t_{0}<T$, then, from (17) and (27), $v>0$ and $q=0$ on $\left.] t_{0}, T\right]$. For $0<t_{0}$, we have $v=f=0$ and, from Lemma $1, E>0$ and $q=$ const on $\left.] 0, t_{0}\right]$. If $0<t_{0}<T$, then, by continuity, $E>0$ and $q=$ const on 
] $0, t_{0}+\epsilon[$ for some $\epsilon>0$.

In any case we conclude that $p \equiv q \equiv 0$ for $\lambda_{0}=0$, which is not admitted by the Pontryagin maximum principle. Therefore $\lambda_{0}>0$, and we can assume without loss of generality that $\lambda_{0}=1$.

Lemma 3 : If $s\left(t_{0}\right)=0$ with $0<t_{0} \leq T$, then $s(t)=0$ for all $t \in\left[t_{0}, T\right]$.

Proof: Suppose that the hypotheses of the lemma are satisfied, but that $s(\tau) \neq 0$ for some $\tau$ with $t_{0}<\tau<T$. Since $s\left(t_{0}\right)=s(T)=0$, it follows by Lemma 2 that $s>0$ on an interval $] t_{1}, t_{2}$ [, with $t_{0} \leq t_{1}<t_{2} \leq T$, and $s\left(t_{1}\right)=s\left(t_{2}\right)=0$. From (20) and Corollary 2 , we have $f=1, E>0$ and $q=$ const on $] t_{1}, t_{2}[$. The function $s$ has a minimum at $t_{1}$, and it is of class $C^{1}$ in a neighbourhood of $t_{1}$, since $E\left(t_{1}\right)>0$ by Lemma 1. Therefore $s^{\prime}\left(t_{1}\right)=0$, and it follows from (23) that

$$
s(t)=2(q-1)\left[\cosh \left(t-t_{1}\right)-1\right]
$$

for $t \in\left[t_{1}, t_{2}\right]$. This violates $s>0$ on $] t_{1}, t_{2}\left[\right.$ and $s\left(t_{2}\right)=0$

Lemma 4 : Suppose that the switching function vanishes locally.

(i) On an interval where $v>0$ and $s=0$, we have

$$
q^{\prime} v=2(p-1)=2(q v-1) \text { and } p \leq 1
$$

(ii) On an interval where $E>0$ and $s=0$, we have

$$
p=q v=1 \text { and } \quad v=f=q^{-1}=\text { const } .
$$

Proof: The function $q$ is differentiable when $q=$ const and also when $s=p-q v$ $=0$ and $v>0$, since $q=p v^{-1}$ in this case. Differentiating the equation $p-q v=0$ and using (19) and (3) yields (28), whence we deduce, since $q$ is non-increasing, that $p \leq 1$.

For $E>0$, we have from $q=$ const that $q^{\prime}=0$, thus we obtain $p=1$ and $v=q^{-1}$ from (28). The equation $v^{\prime}=0$ implies $f=v$

Corollary 4: We cannot have both $E>0$ and $s=0$ in a neighbourhood of 0 or of $T$.

Proof : Suppose that $E>0$ and $s=0$ in a left neighbourhood of $T$. Then Lemma 4 (ii) implies that $q v=1$ in that neighbourhood. Since $q$ is continuous from 
the left, we would have $q(T) v(T)=1$, but $q(T)=0$ by Corollary 1 .

The proof for a right neighbourhood of 0 is similar and uses $v(0)=0$

Lemma 5 : If $E\left(t_{0}\right)=0$ with $0<t_{0} \leq T$, then $E(t)=0$ for all $t \in\left[t_{0}, T\right]$.

Proof : We first note, by Corollary 2 and Lemma 3, that $s=0$ on $\left[t_{0}, T\right]$.

If $E$ does not vanish on the whole interval $\left[t_{0}, T\right]$, then $E>0$ on some open interval ] $t_{1}, t_{2}$ [, for $t_{0} \leq t_{1}<t_{2} \leq T$, with $E\left(t_{1}\right)=0$ and either $E\left(t_{2}\right)=0$ or $t_{2}=T$. Since the case $t_{2}=T$ is excluded by Corollary 4 , we have $t_{2}<T$ and $E\left(t_{1}\right)=E\left(t_{2}\right)=$ 0 . Also, by Lemma $4(i i), v=f=$ const and hence, by (4), $E^{\prime}=$ const on $] t_{1}, t_{2}[$, and we conclude that $E=0$ on $\left[t_{1}, t_{2}\right]$ in contradiction to our assumption that $E>0$ on ]$t_{1}, t_{2}[$.

Therefore we see that our assumption was wrong and that $E=0$ on $\left[t_{0}, T\right]$

Corollary 5 : There exist $T_{1}$ and $T_{2}$, with $0<T_{1} \leq T_{2} \leq T$, such that $s>0$ on ] $0, T_{1}\left[\right.$ and $s=0$ on $\left[T_{1}, T\right]$, and such that $E>0$ on $] 0, T_{2}\left[\right.$ and, if $T_{2}<T, E=0$ on $\left[T_{2}, T\right]$.

Proof : Since $v(0)=0$, we have, by (4), that $E^{\prime}>0$ in a neighbourhood of 0 . Therefore $E>0$ on an interval $] 0, T_{2}\left[\right.$, with $0<T_{2} \leq T$, and $E\left(T_{2}\right)=0$, if $T_{2}<T$. By Lemma $5, E=0$ on $\left[T_{2}, T\right]$, if $T_{2}<T$.

Let $T_{1}=\inf \{t: s=0$ on $[t, T]\}$. Then $s=0$ on $\left[T_{1}, T\right]$. By the foregoing and by Corollary 4, we must have $0<T_{1}$. By Lemma $3, s>0$ on $] 0, T_{1}[$. Finally, we have $T_{1} \leq T_{2}$ by Corollary 2

We now distinguish four possibilities how the inequalities $0<T_{1} \leq T_{2} \leq T$ can be satisfied. From Corollary 5 , they lead to the following behaviour of $E$ and $s$ :

(a) $0<T_{1}=T_{2}=T$ :

$E>0, s>0$ and $f=1$ on $] 0, T$.

(b) $\quad 0<T_{1}=T_{2}<T$ :

$E>0, s>0$ and $f=1$ on $] 0, T_{1}[$,

$E=s=0$ on $\left[T_{1}, T\right]$.

(c) $0<T_{1}<T_{2}<T$ :

$E>0, s>0$ and $f=1$ on $] 0, T_{1}[$,

$E>0, s=0$ and, by Lemma $4(i i), v=$ const $=v_{1}$ on $\left[T_{1}, T_{2}[\right.$,

$E=s=0$ on $\left[T_{2}, T\right]$. 
(d) $\quad 0<T_{1}<T_{2}=T$ :

$$
\begin{aligned}
& E>0 \text { and } s>0 \text { on }] 0, T_{1}[, \\
& E>0 \text { and } s=0 \text { on }\left[T_{1}, T[.\right.
\end{aligned}
$$

By Corollary 4 the case $(d)$ cannot occur. In cases $(a),(b)$ and $(c)$ we shall show that the following necessary conditions hold:

(a) $T \leq T_{0}$

(b) $\quad T_{0}<T \leq T_{c}$ and $T_{1}=T_{2}=T_{0}$.

(c) $\quad T>T_{\mathrm{c}}$ and the relation (5).

Recalling from Section 2(iii) that $v_{1}, T_{1}$ and $T_{2}$ are determined uniquely by (5) in case $(c)$, we have thus proved the theorem.

It is obvious from the definition of $T_{0}$ that $T \leq T_{0}$ in case $(a)$ and $T_{1}=T_{2}=T_{0}$ $<T$ in case $(b)$. For the proof of the remaining assertions we need

Lemma 6 : Suppose that $E=s=0, \vec{v} \neq \dot{\sigma}$ on $\left[T_{2}, T\right]$. Then, for $t \in\left[T_{2}, T\right]$,

$$
p(t)=\frac{2 v(t)[v(t)-v(T)]}{v^{2}(t)-\sigma^{2}} \leq 1
$$

Proof: By Section 2(ii) we can reparametrize by $v>0$. From (11) and (28), it then follows that

$$
\frac{\mathrm{d}}{\mathrm{d} v}\left[q\left(v^{2}-\sigma^{2}\right)\right]=\frac{q^{\prime}}{v^{\prime}}\left(v^{2}-\sigma^{2}\right)+2 q v=2
$$

We conclude that

$$
q=\frac{2(v-c)}{v^{2}-\sigma^{2}}
$$

where $c=v(T)$ in order to satisfy (24). As $p=q v$, we obtain (30). That $p \leq 1$ has already been shown in Lemma $4(i)$

We now show that $T \leq T_{\mathrm{c}}$ for $v_{0}>\sigma$ in case $(b)$. By comparing (14) where we set $T_{2}=T_{0}$ and $v_{1}=v_{0}$, 


$$
T=T_{0}+\frac{1}{2} \log \frac{v_{0}^{2}-\sigma^{2}}{v^{2}(T)-\sigma^{2}}
$$

and (6), we see that $T \leq T_{\mathrm{c}}$ is equivalent to

$$
\frac{v_{0}^{2}+\sigma^{2}}{2 v_{0}} \leq v(T)
$$

Since $E=s=0$ on $\left[T_{0}, T\right]$, we have from Lemma 6 that

$$
p\left(T_{0}\right)=\frac{2 v_{0}\left(v_{0}-v(T)\right)}{v_{0}^{2}-\sigma^{2}} \leq 1,
$$

which gives (31).

For the case $(c)$, we have seen in Section 2(iii) that $\sigma<v_{1}<v_{0}$ and $T=\tau\left(v_{1}\right)$ with $\tau$ given by (15). Since $\tau$ is decreasing, we conclude that $T=\tau\left(v_{1}\right)>\tau\left(v_{0}\right)=T_{\mathrm{c}}$. Combining Lemma $4(i i)$, which asserts that $p=1$ on $\left[T_{1}, T_{2}\right]$, with Lemma 6 , we infer that

$$
p\left(T_{2}^{*}\right)=\frac{2 v_{1}\left(v_{1}-v(T)\right)}{v_{1}^{2}-\sigma^{2}}=1
$$

which gives (5).

\section{REFERENCES}

[1] BEHNCKE, H.: Optimization models for the force and energy in competitive oports. Math. Meth. in the Appl. Sci. 9 (1987), $298-311$.

[2] KELLER, J.B.: A theory of competitive running. Physics Today 26 (1973), $42-47$.

[3] KELLER, J.B.: Optimal velocity in a race. Amer. Math. Monthly 81 (1974), $474-480$.

[4] IOFFE, A.D. and V.M. TIHOMIROV: Theory of Extremal Problems. Amsterdam. New York Oxford: North Holland 1979. 